\title{
Assessment of left ventricular diastolic function by MR: why, how and when
}

\author{
Ricardo Duarte · Gabriel Fernandez
}

Received: 21 April 2010 / Accepted: 11 May 2010/Published online: 15 June 2010

(C) European Society of Radiology 2010

\begin{abstract}
Cardiovascular magnetic resonance (CMR), a valuable non-invasive technique for the evaluation of the cardiovascular system, has already been accepted as the "gold standard" for the assessment of systolic function. The assessment of diastolic function is important not only for diagnosis purposes, but also in terms of prognosis. ECG-triggering phase-contrast (PC) CMR allows the routine assessment of diastolic function by measuring the transmitral and pulmonary venous flow with high accuracy and reproducibility, using morphological and quantitative parameters similar to those obtained by transthoracic echocardiography, which are so familiar to general cardiologists. Therefore, the increasing role of CMR in the assessment of the cardiovascular system requires a greater awareness and knowledge of this condition by radiologists. The aim of this study is to review the main mechanisms and common causes of left ventricle diastolic dysfunction, provide a practical approach for the assessment of LV diastolic function and illustrate the different degrees of diastolic dysfunction.
\end{abstract}

Keywords Cardiovascular magnetic resonance (CMR) . Systolic function $\cdot$ Diastolic function $\cdot$ Measurement

\footnotetext{
R. Duarte $(\square)$

Department of Radiology, Centro Hospitalar Gaia/ Espinho,

Vila Nova de Gaia, Portugal

e-mail: guerra.duarte@gmail.com

G. Fernandez

Department of Radiology, Hospital Povisa,

Vigo, Spain

e-mail: gabriel.fdez.perez@gmail.com
}

\section{Introduction}

Congestive heart failure (CHF) is the third most prevalent form of heart disease with 550,000 new cases being reported annually [1]. CHF caused by a predominant abnormality in diastolic function has been increasingly recognised as a common entity and a cause of significant morbidity and mortality, as $40-50 \%$ of the patients with heart failure may have isolated diastolic dysfunction with a normal or near normal left ventricle (LV) systolic function [2-4].

Moreover, in the last 2 decades, the proportion of patients with $\mathrm{CHF}$ and normal LV systolic function has increased considerably, from $38 \%$ to $54 \%$ [5].

Primary diastolic dysfunction is usually seen in patients with hypertension, valvular heart disease and hypertrophic, restrictive or ischaemic cardiomyopathy [6].

An early diagnosis is decisive in order to prevent irreversible structural alterations and systolic dysfunction. Distinguishing diastolic from systolic heart failure is crucial, because the treatment for one may exacerbate the other, and with proper treatment the prognosis of diastolic dysfunction is more favourable than that of systolic dysfunction [7, 8].

In this paper, after discussing the mechanisms and common causes of LV diastolic dysfunction, we provide a practical approach for the assessment of LV diastolic function, focusing on the use of phase-contrast imaging (PC) in the evaluation of transmitral and pulmonary vein flow, and illustrate the different degrees of diastolic dysfunction.

\section{Mechanisms of diastolic dysfunction}

Conventionally, diastole can be divided into four phases: isovolumetric relaxation, left ventricle (LV) rapid early filling, diastasis and LV late filling or atrial contraction [9]. 
Diastolic dysfunction results from myocardial relaxation and ventricular compliance abnormalities that lead to an increase in LV end-diastolic pressure. Myocardial relaxation is an active or ATP-consuming process that affects the isovolumetric relaxation phase and part of the rapid early filling phase, while ventricular compliance is a passive process that influences all three filling phases of diastole.

The mechanisms responsible for the abnormalities in diastolic function can be due to factors intrinsic to the myocardium itself (myocardial) or factors that are extrinsic to the myocardium (extramyocardial) [3]. Myocardial factors include changes in calcium homeostasis, energetics (e.g., changes in the ADP/ATP ratio and ADP and phosphate concentration), myofilaments, cytoskeleton, neurohumoral and cardiac endothelial activation, as well as increases in the content of extramyofilament cytoskeletal proteins $[3,10]$.

Diastolic CHF represents the clinical entity, characterised by signs and symptoms due to increased extravascular water and decreased tissue/organ perfusion, in which patients with CHF have normal or near normal systolic function and significant diastolic dysfunction [10].

\section{Diagnosis and parameters for the assessment of diastolic function}

Despite the high prevalence of diastolic dysfunction, the diagnosis remains challenging, as differentiation between systolic and diastolic CHF cannot be made based on the clinical history, physical examination or ECG, as both have similar changes [2], and so the diagnosis of diastolic dysfunction requires the demonstration of elevated filling pressures. For this reason diagnostic criteria based on measurements of systolic and diastolic function have been developed by The Working Group for the European Society of Cardiology [11].

Because invasive cardiac catheterisation to demonstrate elevated filling pressures is not feasible for routine use, non-invasive techniques have been developed to assess diastolic function, based on two biomechanical indices: left ventricle (LV) relaxation and compliance, which are inferred by measuring the transmitral inflow data with corroborating pulmonary venous flow data $[11,12]$. Transthoracic echocardiograph (TTE) is the most important tool in the evaluation of diastolic function. Nevertheless, this technique has some limitations: limited field of view, cosine $\theta$ errors relative to the flow direction and an inadequate acoustic window in approximately $15-20 \%$ of patients [13].

Cardiovascular magnetic resonance (CMR) imaging is a non-invasive technique that, on account of its high spatial and temporal resolution, excellent image quality and unique potential of three-dimensional function analysis with great accuracy and reproducibility, is already accepted as the "gold standard" for the assessment of LV morphology and systolic function [14].

Recent technical developments of ECG-triggering PCCMR allow the routine assessment of diastolic function by measuring the transmitral and pulmonary venous flow and evaluating the velocity vs. time curves generated, which reflect the filling patterns in a similar way to the methods used in TTE $[15,16]$.

\section{CMR imaging}

Basics and feasibility of phase-contrast imaging

Phase-contrast CMR is a validated and accurate noninvasive technique for the evaluation of velocity, volume and pattern of blood flow [13, 17-24]. This technique is based on the shift that moving spins of intravascular protons acquire when flowing along a magnetic field gradient relative to stationary spins, which is directly proportional to their velocity along the direction of the gradient, allowing velocity encoding of moving structures and blood in any chosen direction and the calculation of velocities of the myocardium and blood flow $[15,25,26]$. Phase-contrast sequences are able to encode the velocity perpendicular (through-plane) or parallel (in-plane) to the direction of flow $[15,25,26]$. Two images are reconstructed, the magnitude "image", which provides anatomical information, and the velocity-encoding map "image", which provides flow information.

The value of the velocity encoding (VENC) corresponds to the amplitude of the magnetic gradient and is given in centimetres per second [15]. VENC is determined by the user and establishes the highest and lowest detectable velocity encoded; the closer it is to the maximum expected velocity in the region of interest, the more precise the measurement becomes $[15,25,26]$. If the VENC is set below the peak velocity in the region of interest, aliasing or velocity wraparound will occur $[15,25,26]$.

Pressure gradients can also be calculated using the modified Bernoulli equation: Pressure Gradient $(\mathrm{mmHg})=$ $4 \times \mathrm{V}_{\max }{ }^{2}$, where $\mathrm{V}_{\max }$ is the peak velocity $(\mathrm{m} / \mathrm{s})$ [15].

The advantage of PC-CMR over TTE is that the first quantifies the volume flow over the entire region (i.e., the valve area) as opposed to TTE in which the flow and velocity are assessed in a relatively small area, potentially producing sample volume errors because the flow is not homogeneous over such a region. Another advantage of PC-CMR is that because of its high temporal and spatial resolution potential, cosine $\theta$ errors can easily be avoided and flow can be measured with a higher level of accuracy. 
In recent studies, PC-CMR flow measurement revealed lower peak velocities in comparison to Doppler echocardiography [13, 27-31]. Nevertheless, it has been demonstrated that the measurement of flow by PC-CMR is more accurate than by Doppler echocardiography, as in the case of Doppler, the flow is assessed in a relatively small vessel area and assumes a constant velocity over the whole vessel area, while in PC-CMR the volume flow is evaluated over the entire vessel area, explaining why Doppler tends to overestimate peak velocities by as much as $25 \%[13,32,33]$.

How to image the transmitral flow and the pulmonary venous flow

At our institution, all CMR examinations are performed in a 1.5-T MR unit (Achieva, Philips Healthcare, The Netherlands) with a five-element phased array cardiac dedicated coil.

Localiser images are obtained in all three planes, and interactive real-time images are acquired to plan the optimal cardiac planes, specifically the LV short axis, the LV vertical long axis, the $\mathrm{LV}$ horizontal long axis and $\mathrm{LV}$ outflow. Cine images are obtained using ECG-triggering steady-state sequence (balanced fast field echo) in the different cardiac planes in order to precisely plan the mitral valve plane. Transmitral flow data, including peak velocities and volume flow of $\mathrm{E}$ and $\mathrm{A}$ waves, are assessed at rest and under the Valsalva manoeuvre (performed with forced exhalation against a closed airway) in an imaging plane parallel to the mitral annular plane at the level of the mitral valve leaflet tips (Fig. 1).

Right superior pulmonary venous flow data, including peak velocities and volume flow of S, D and AR waves, are assessed in an imaging plane perpendicular to the pulmonary vein, $1 \mathrm{~cm}$ away from the junction of the pulmonary vein with the left atrium (Fig. 2).

Patients are imaged using retrospective ECG-triggering PC-CMR sequences in order to collect data continuously throughout the entire cardiac cycle, acquiring 40 cardiac phases per cycle. Technical parameters are as follows: TR $4.1 \mathrm{~ms} / \mathrm{TE} 2.5 \mathrm{~ms}$, section thickness of $10 \mathrm{~mm}$, flip angle of $15^{\circ}$ and a temporal resolution between 16 and $28 \mathrm{~ms}$.

Fig. 1 PC-CMR of transmitral inflow for assessment of diastolic function. Cine four-chamber (a) and LV vertical axis (b) showing the orientation of through-plane PC-CMR imaging (dashed line).

(c) Magnitude and

(d) phase-contrast images are obtained in a plane parallel to the mitral annular plane at the level of the mitral valve leaflet tips. The contour of the region of interest is drawn at the level of the leaflet tips and includes the whole cross-section of the transmitral inflow (red circle). $L V$, left ventricle; $L A$, left atrium; $R V$, right ventricle; $R A$, right atrium
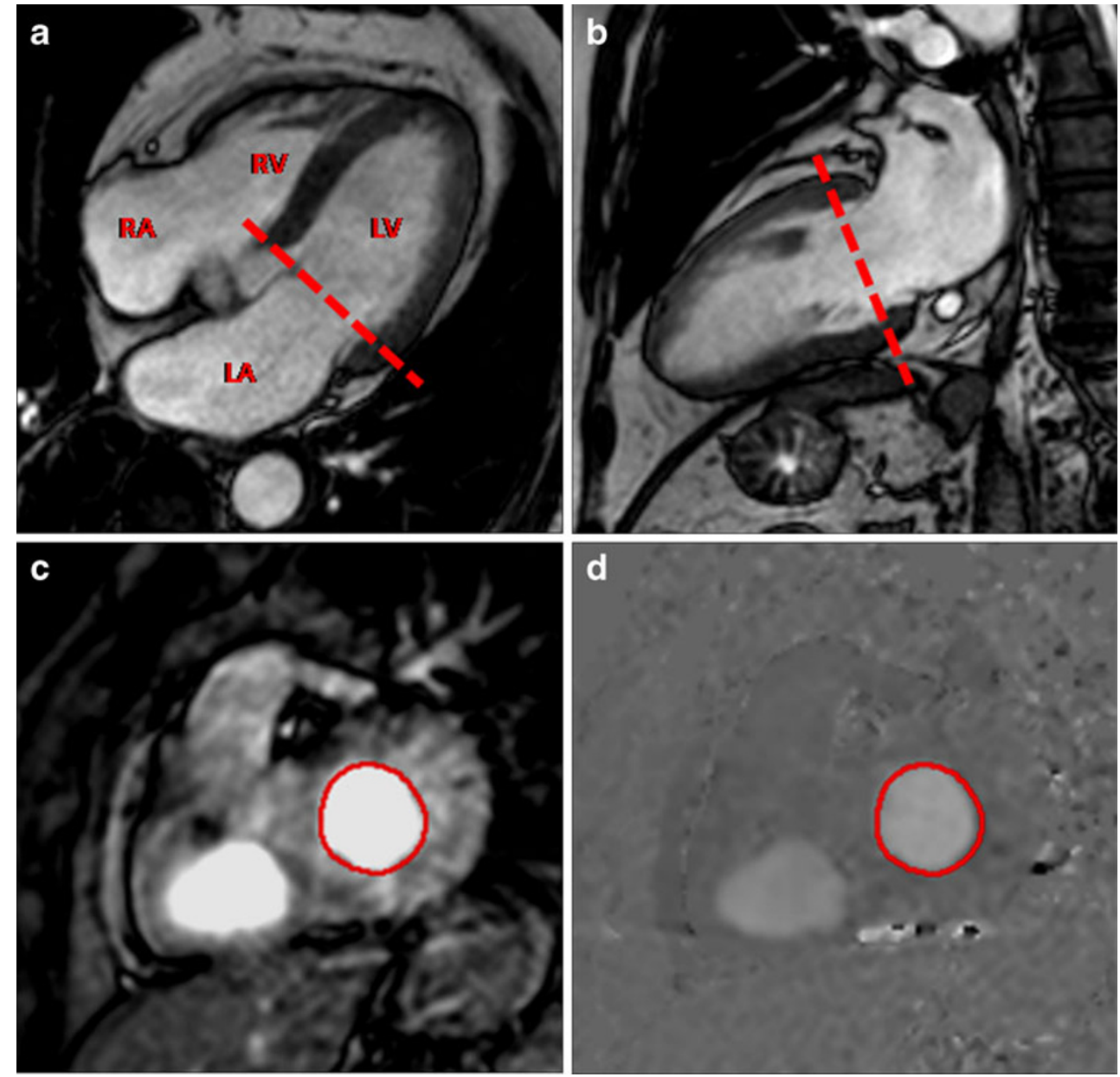
Fig. 2 PC-CMR of pulmonary venous flow for assessment of diastolic function. Axial (a) and coronal (b) survey demonstrating the orientation of throughplane PC-CMR imaging (dashed line). (c) Magnitude and (d) phase-contrast images are obtained in a plane perpendicular to the right superior pulmonary vein (arrow), $1 \mathrm{~cm}$ away from the junction of the pulmonary vein with the left atrium. $R P A$, right pulmonary artery; $R P V$, right superior pulmonary venous
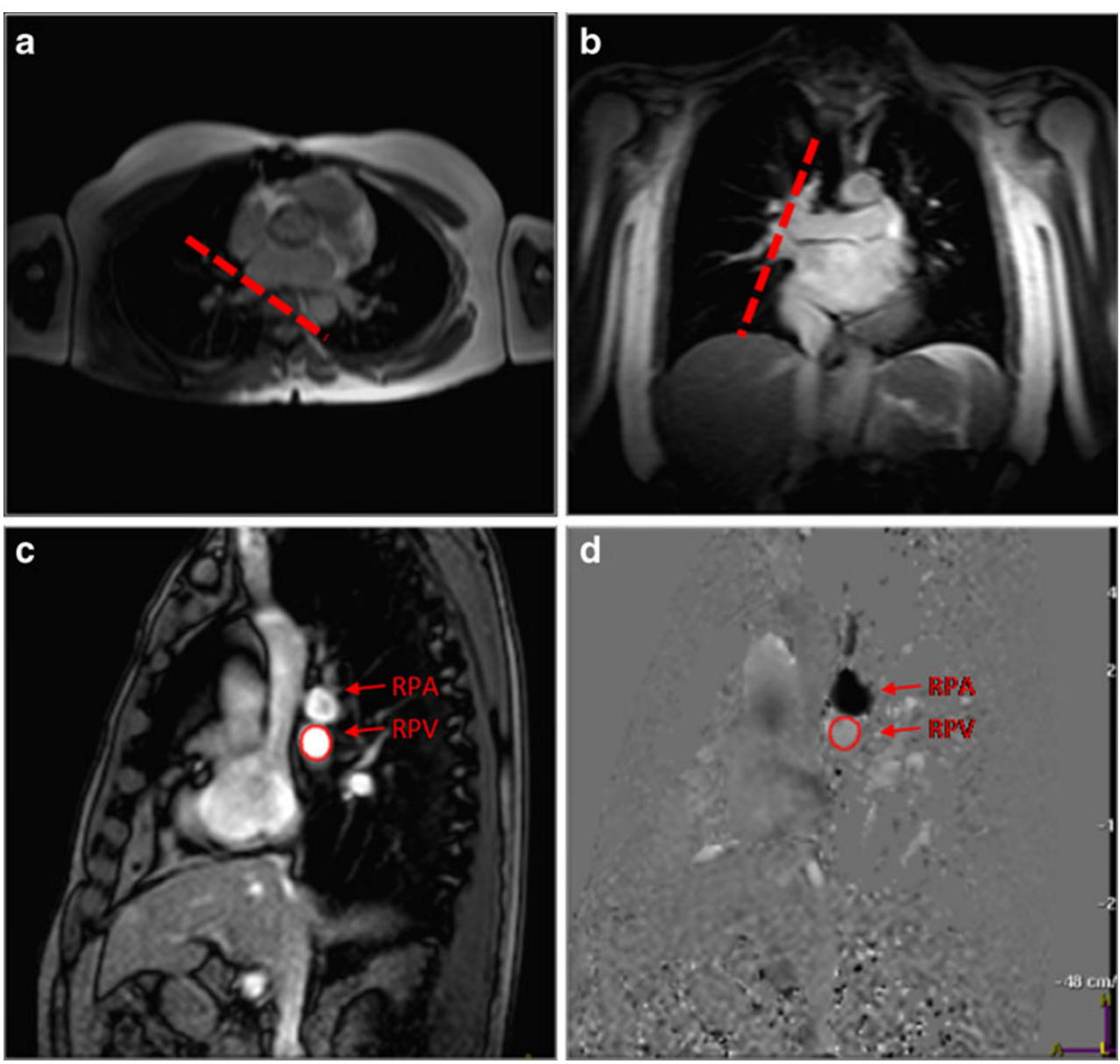

VENC is set at $90 \mathrm{~cm} / \mathrm{s}$ for the transmitral flow and at $100 \mathrm{~cm} / \mathrm{s}$ for the pulmonary vein flow. PC-CMR acquisition times range from 8 to $15 \mathrm{~s}$, depending on the heart rate. Some limitations must be pointed out: first, as is the case with the TTE, CMR diastolic flow indices are influenced by arrhythmias, pressure gradients, the heart rate and the hydration status of the patient, independent of the technique used; second, in addition to the inadequate VENC setting already mentioned, other sources of error in PC-CMR measuring include motion of the imaging plane during data acquisition due to cardiac or respiratory motion, inadequate temporal and spatial resolution, and field heterogeneity, which could potentially be improved by using 3D PC-CMR [15].

\section{Flow analysis}

The PC-CMR images obtained are sent to a dedicated 3D workstation (Philips, The Netherlands), and flow analysis is performed by a radiologist using flow-dedicated software (ViewForum Workstation R5.1; Philips Healthcare, The Netherlands). The contours in the regions of interest (ROIs) are manually drawn and automatically copied from magnitude to velocity images and vice versa. Whenever necessary, the ROI shape and position are adapted to the region of interest for each phase of the cardiac cycle. Measurements of the

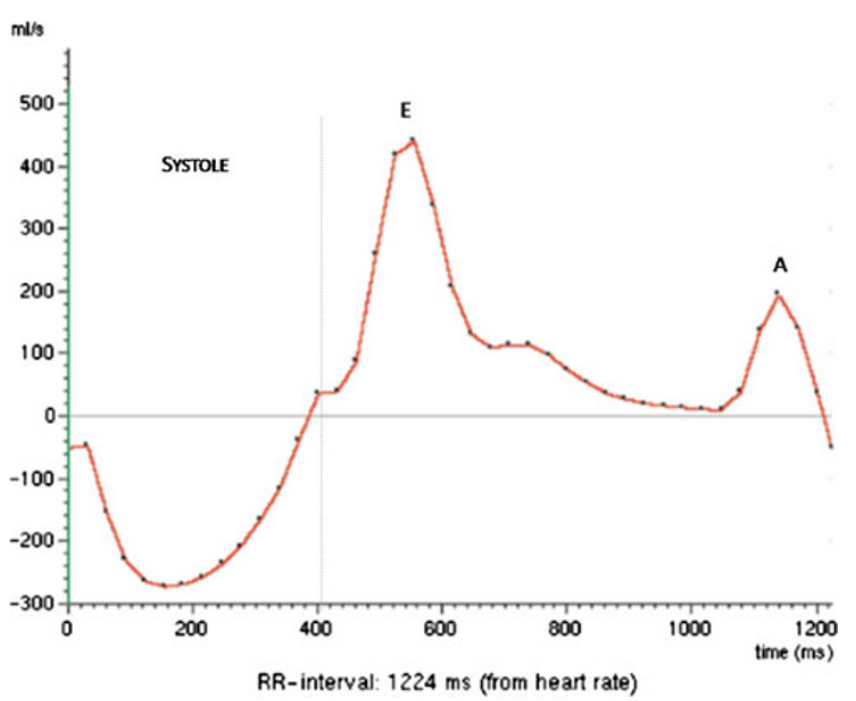

Fig. 3 Normal transmitral inflow pattern. E, initial rapid early flow; $A$, late or atrial flow 


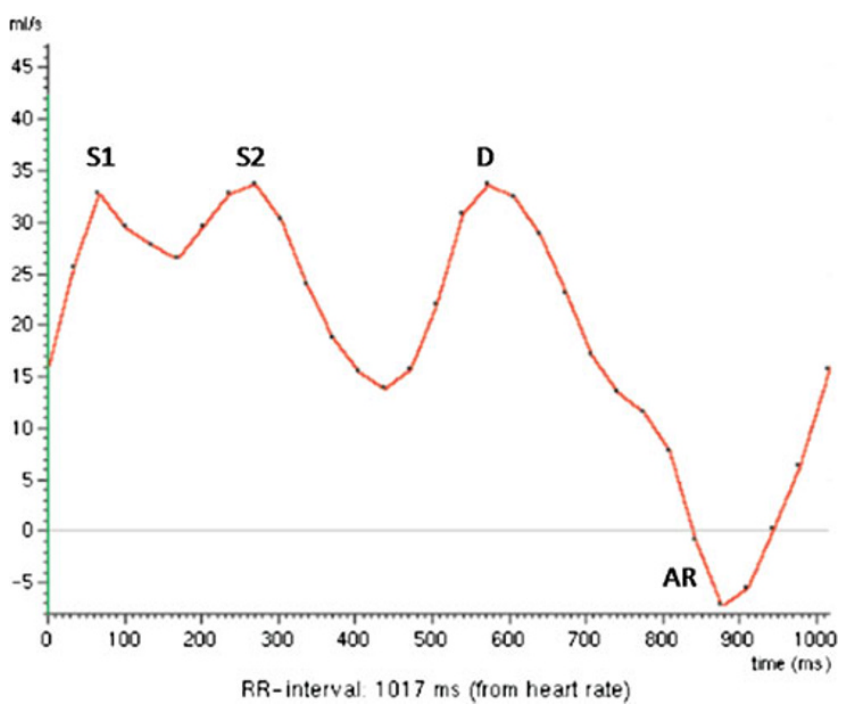

Fig. 4 Normal pulmonary venous inflow pattern. S1, first systolic peak; $S 2$, second systolic peak; $D$, diastolic flow; $A R$, atrial reversed

transmitral inflow yielding information on $\mathrm{E}$ and $\mathrm{A}$ waves and on pulmonary venous flow and peak velocities of S, D and AR waves are displayed on a velocity or flow vs. time graph. The peak velocity ratio of the $\mathrm{E}$ and $\mathrm{A}$ waves, called the E/A ratio, is calculated. The deceleration time (DT) is also calculated using the method described by Appleton et al. [34].

Normal transmitral and pulmonary venous flow pattern

The normal transmitral inflow curve is characterised by an initial rapid early flow (E wave) and a late or atrial flow (A wave). As the $E$ wave is higher than the $A$ wave $(E>A)$, the E/A ratio is greater than 1 (Fig. 3).

The normal pulmonary venous flow curve consists of one or two systolic peaks and one diastolic peak, with equivalent flow velocities, plus a reversed flow in end diastole (Fig. 4).

The systolic (S) flow corresponds to flow into the left atrium (LA) during ventricular systole and consists of one or two peaks; the first systolic peak (S1) is caused by the reduction of the LA pressure due to LA relaxation and longitudinal LV shortening with subsequent motion of the annulus of the atrioventricular valve, and the second systolic peak (S2) reflects right ventricular stroke volume and atrial compliance. The diastolic (D) flow represents the reduction of the LA pressure during ventricular filling in early diastole. The reversed flow is caused by atrial contraction and is called atrial reversed

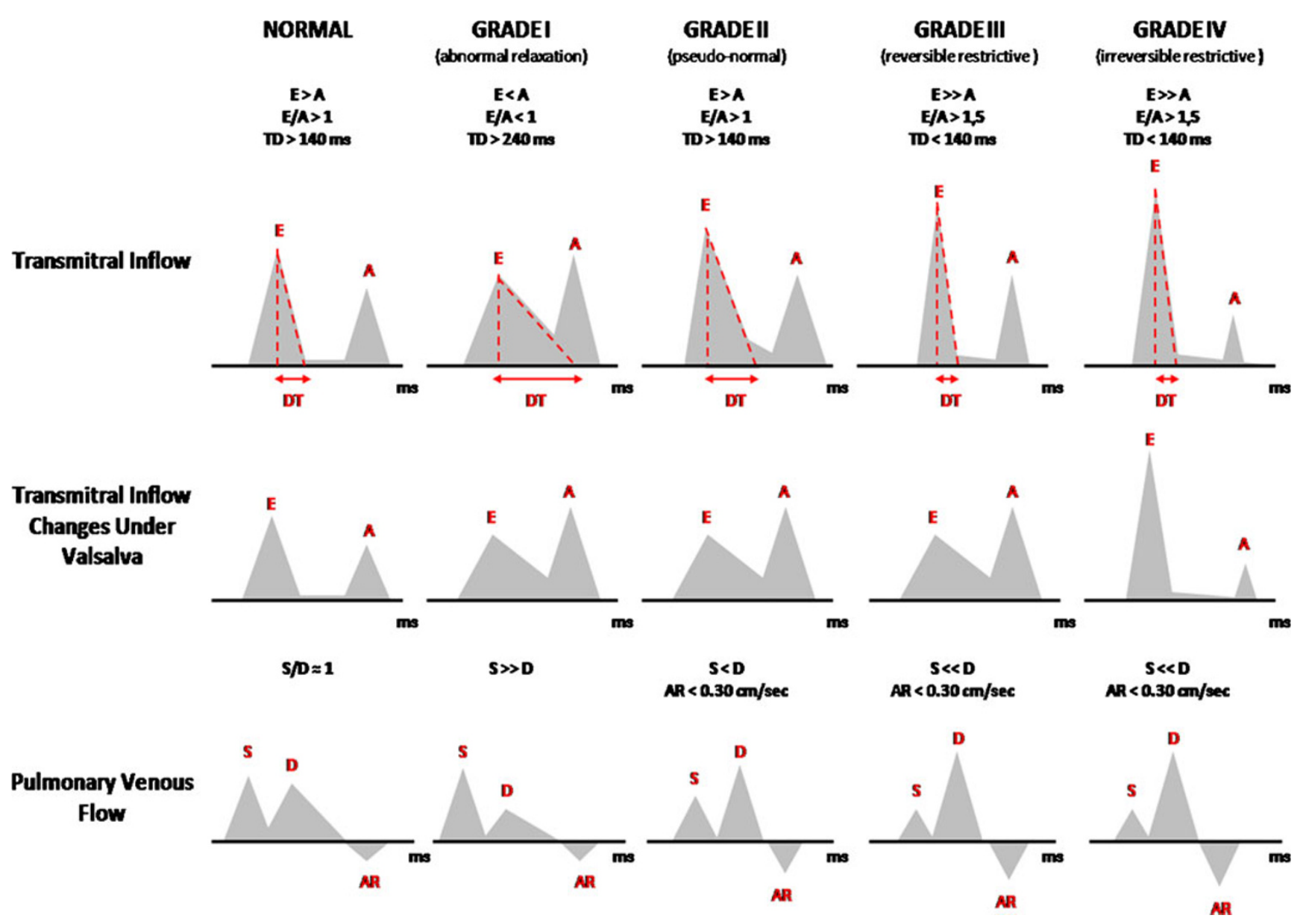

Fig. 5 Classification of diastolic dysfunction grades (I-IV) 
(AR). The normal AR wave velocity is less than $0.30 \mathrm{~cm} / \mathrm{s}$.

Diastolic dysfunction patterns

Diastolic dysfunction can be classified in four grades [35] (Fig. 5).

In grade I there is an abnormal relaxation, which is characterised by a transmitral inflow pattern with an $\mathrm{A}$ wave higher than the $\mathrm{E}$ wave $(\mathrm{E}<\mathrm{A})$ and an $\mathrm{E} / \mathrm{A}$ ratio less than 1; prolonged deceleration time (DT) is commonly associated (Fig. 6).

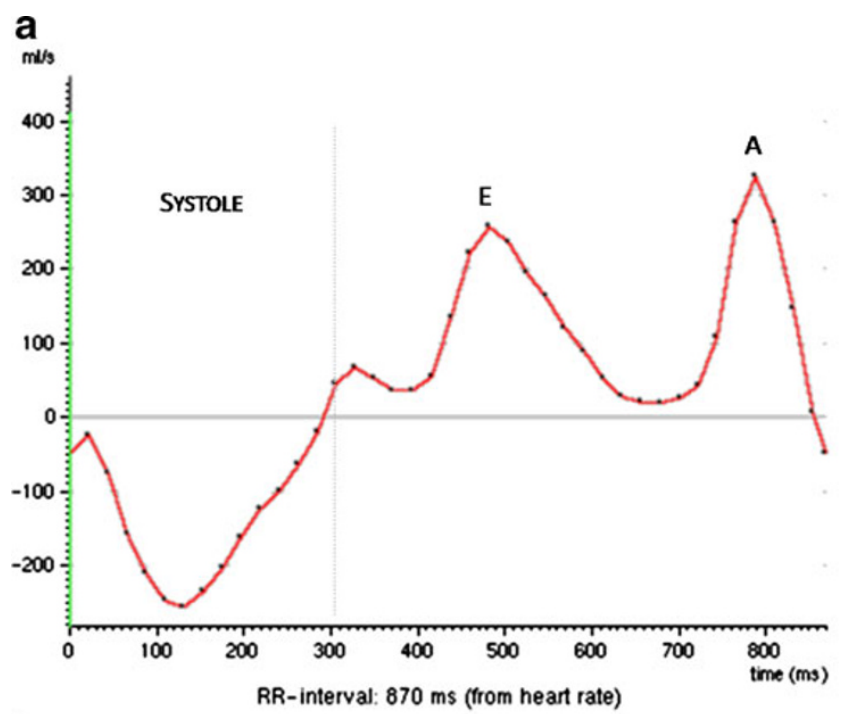

b

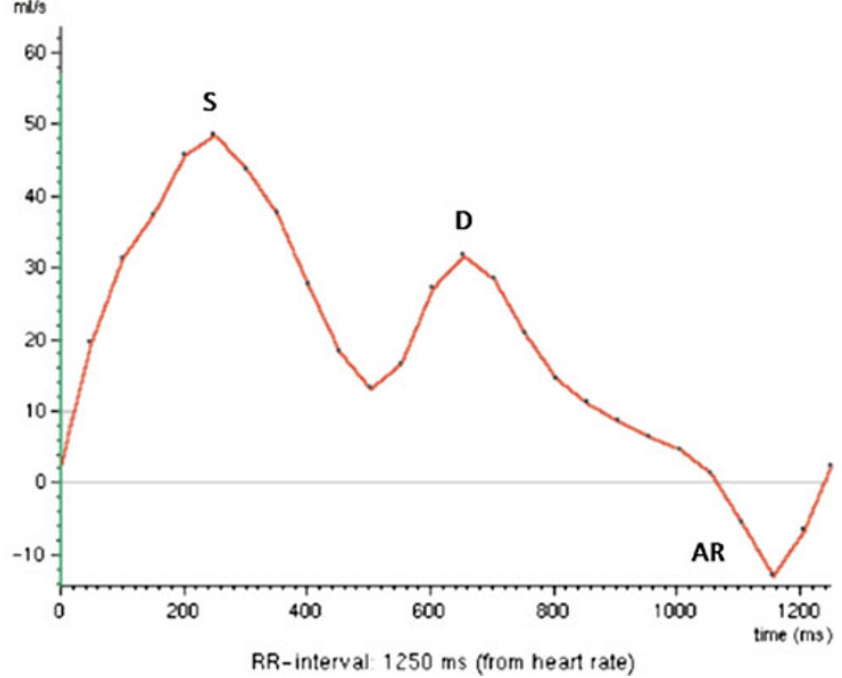

Fig. 6 A 63-year-old woman with a long-standing history of hypertension and normal systolic function. (a) Transmitral inflow analysis shows an $\mathrm{E}$ wave (E) lower than the A wave (A) and an E/A ratio $<1$ due to reduced LV filling in early diastole indicating a grade I diastolic dysfunction (abnormal LV relaxation). (b) Pulmonary venous flow analysis demonstrates a systolic flow peak (S) higher than the diastolic (D) with normal reversed flow (AR)
As LV relaxation becomes further compromised and the LV telediastolic pressure increases, the contribution of atrial contraction to the LV filling is once again reduced, resulting in a transmitral inflow pattern similar to normal, as the $\mathrm{E}$ wave is again higher than the $\mathrm{A}$ wave $(\mathrm{E}>\mathrm{A})$, and the $\mathrm{E} / \mathrm{A}$ ratio becomes greater than 1 . This transmitral inflow pattern is termed pseudo-normal or grade II (Fig. 7).

The Valsalva manoeuvre reduces preload and, in patients with normal diastolic function, produces similar reductions in the peak velocities of the $\mathrm{E}$ and $\mathrm{A}$ waves, so the $\mathrm{E} / \mathrm{A}$ ratio will continue to be greater than 1 .

However, in patients with diastolic dysfunction grade II or pseudo-normal pattern, with the Valsalva manoeuvre the pseudo-normal pattern becomes an abnormal relaxation pattern or grade $\mathrm{I}$, and so the E/A ratio will be less than 1, allowing differentiation from the normal pattern.

The assessment of pulmonary venous flow is also useful in differentiating a pseudo-normal pattern from a normal pattern. In the case of a pseudo-normal pattern, the systolic wave is smaller than the diastolic wave $(S<D)$, and the $A R$ wave velocity is usually greater than $0.30 \mathrm{~cm} / \mathrm{s}$. As well as the tissue Doppler, tissue PC-CMR, which can improve the assessment of diastolic function and be particularly useful in assessing pseudo-normalisation, is now also available. However, tissue PC-CMR or myocardial velocity mapping cannot yet be feasibly adopted in the clinical setting because of its limited spatial and temporal resolution [25].

Finally, the increasing ventricular stiffness leads to a restrictive pattern, which is divided into reversible (grade III) and irreversible (grade IV), and is characterised by a further increase in $\mathrm{E}$ wave $(\mathrm{E}>>\mathrm{A})$ with a decrease in DT due to flow into the non-compliant ventricle and a pulmonary venous flow pattern showing an $\mathrm{S}$ wave robustly lower than the $\mathrm{D}$ wave $(\mathrm{S}<<\mathrm{D})$ and an $\mathrm{AR}$ wave velocity greater than $0.30 \mathrm{~cm} / \mathrm{s}$ (Fig. 8). The Valsalva manoeuvre is also very helpful in distinguishing a reversible restrictive pattern (grade III) from an irreversible (grade IV) one, because with this manoeuvre the reversible restrictive pattern can turn into an abnormal relaxation pattern, whereas the irreversible restrictive pattern remains unchanged. A restrictive pattern is associated with poor prognosis, with patients often presenting symptoms and signs of $\mathrm{CHF}$, and is characteristic of restrictive cardiomyopathies and dilated cardiomyopathy with poor LV systolic function.

\section{Common causes of diastolic dysfunction}

Several cardiovascular and systemic diseases can cause LV diastolic dysfunction, as it is the most common hypertensive, ischaemic and hypertrophic cardiomyopathy (Table 1). Long-standing pressure overload, such as in systemic 
Fig. 7 A 50-year-old man with ischaemic cardiomyopathy and preserved systolic function. (a) Transmitral inflow analysis demonstrates an $\mathrm{E}$ wave (E) higher than the A wave (A) and an E/A ratio $>1$. However, with the Valsalva manoeuvre (b) the $\mathrm{E}$ wave becomes lower than an $\mathrm{A}$ wave and an E/A ratio $<1$ indicates grade II diastolic dysfunction or pseudo-normal pattern. (c) Pulmonary venous flow analysis shows a diastolic flow peak (D) higher than the systolic (S) with a prominent reversed flow (AR) indicative of an increase in the left atrial filling pressures

hypertension and aortic valve stenosis, leads to an adaptive LV hypertrophy in order to normalise myocardial wall tension. Nevertheless, diastolic dysfunction can occur even in the absence of LV hypertrophy because of subnormal high-energy phosphate metabolism [6]. For that reason, LV diastolic dysfunction can be found in only $25 \%$ of asymptomatic hypertensive patients without LV hypertrophy but in $90 \%$ of those with LV hypertrophy [6].

Ischaemic cardiomyopathy is also a common cause of diastolic dysfunction and is found in approximately $60 \%$ of all patients with acute myocardial infarction [6]. This results from several mechanisms, such as calcium overload of the myocytes with prolonged and incomplete relaxation, LV asynchrony, papillary muscle dysfunction with valvular incompetence, LV myocardial stunning, hibernation and post-infarction remodelling $[6,16]$. Hypertrophic cardiomyopathy is considered the prototype for diastolic heart failure. Relaxation abnormalities in hypertrophic hearts are due to altered myocardial high-energy phosphate metabolism and changes in the passive elastic properties of the myocardium, such as non-uniform myocardial hypertrophy and fibrosis $[6,16]$.

In patients with infiltrative cardiomyopathies, diastolic dysfunction is commonly the primary myocardial disorder and is characterised by increased LV wall thickness and reduced LV compliance, often evolving to a restrictive pattern.

\section{Prognosis of diastolic dysfunction}

Several studies have demonstrated the high morbidity associated with diastolic dysfunction, resulting in frequent hospital admissions with a 1-year readmission rate of close to $50 \%$ in patients with diastolic CHF [36-41], which is comparable to that of patients with systolic CHF [2]. The mortality rate after 5 years is $68 \%$ in patients with diastolic CHF compared with $82 \%$ of patients with systolic CHF [42].

However, any degree of diastolic dysfunction is an important prognostic factor, and it has been demonstrated that in hypertensive patients the abnormal relaxation is associated with an increased risk of cardiovascular events and mortality [42].
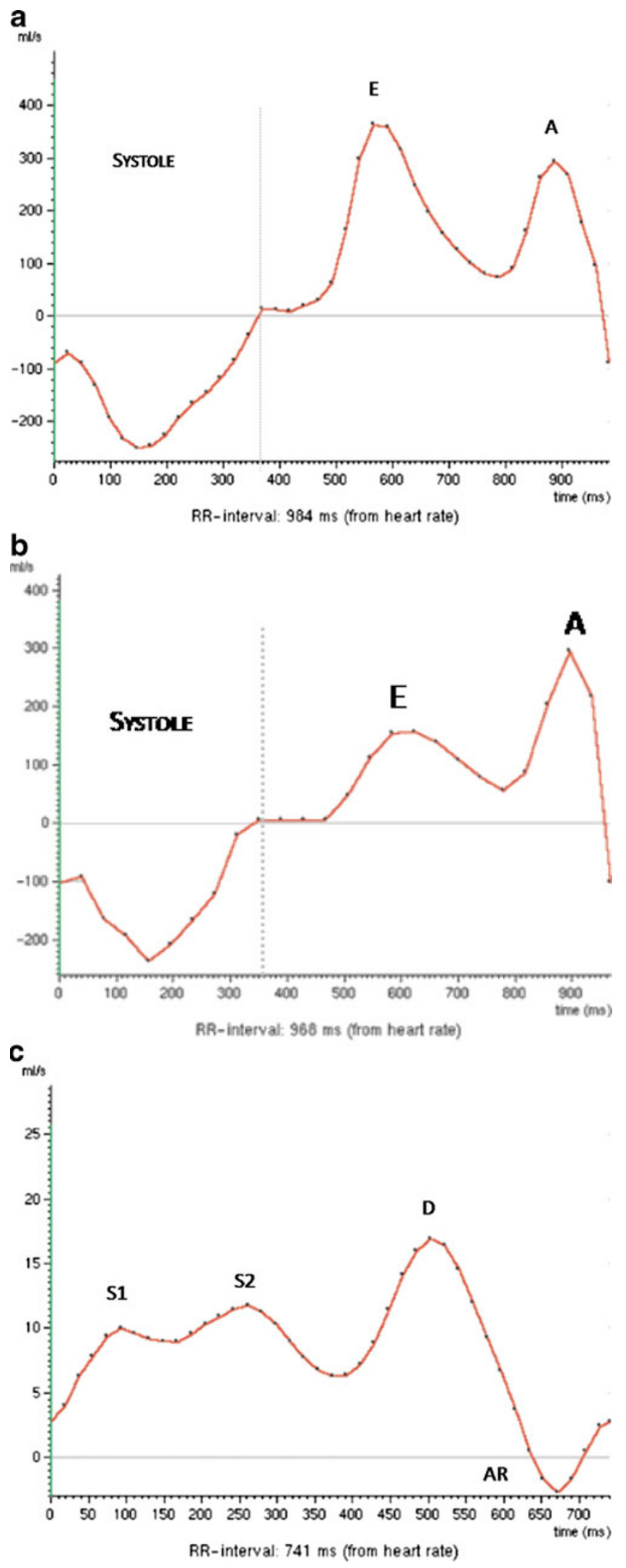


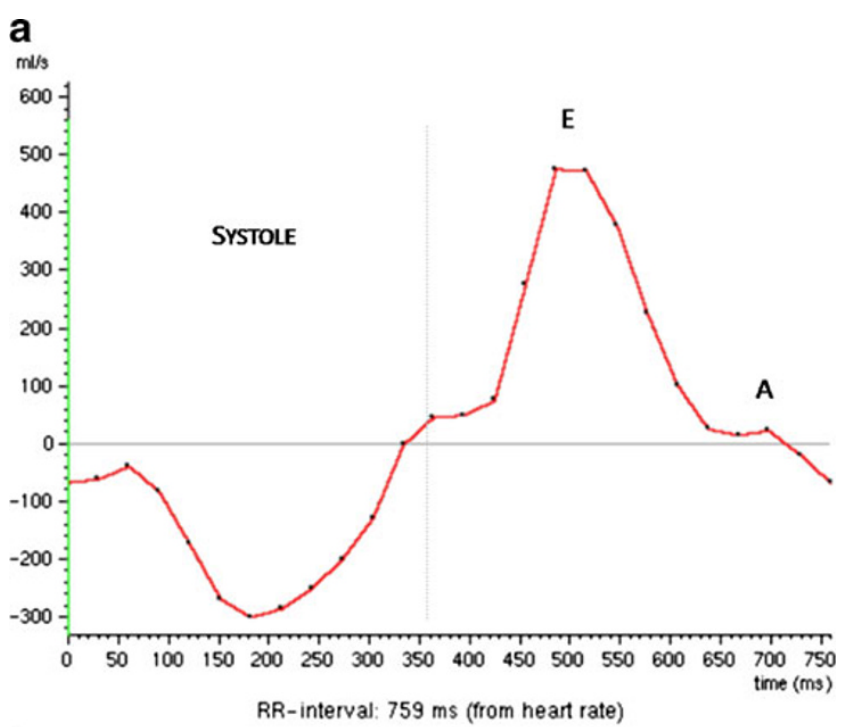

b

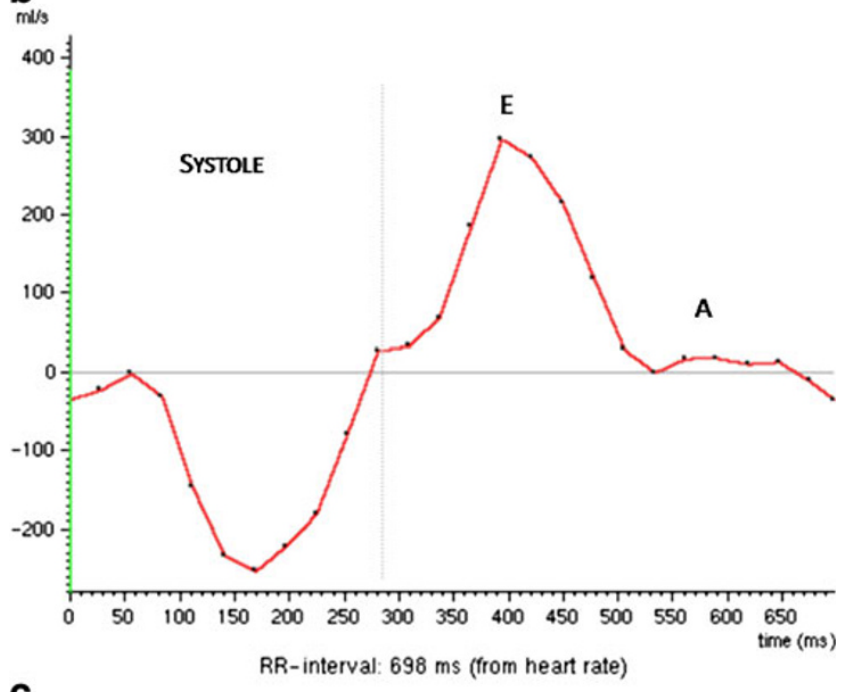

c

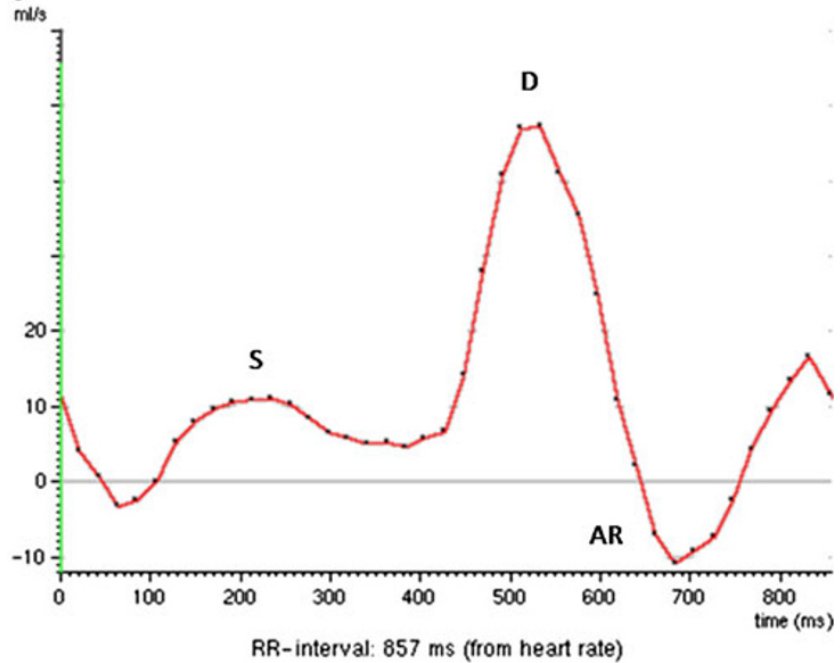

Fig. 8 A 51-year-old man with myocardial iron overload due to haemochromatosis and preserved systolic function. (a) Transmitral inflow analysis shows a prominent $\mathrm{E}$ wave (E) and an A wave (A) that is nearly imperceptible (E/A ratio $>1.5$ ). (b) The mitral flow pattern does not change significantly with the Valsalva manoeuvre. (c) Pulmonary venous flow analysis demonstrates a diastolic flow peak (D) that is notably higher than the systolic (S) with a prominent reversed flow (AR) indicating high left atrial filling pressures

\section{Conclusions}

In clinical practice, diastolic dysfunction is a common entity, especially in patients with hypertension and ischaemic cardiomyopathy, and it is the predominant cause of CHF in $40-50 \%$ of patients.

Cardiovascular magnetic resonance imaging is considered the "gold standard" for evaluating the morphology and LV systolic function, and is widely used in the evaluation of several cardiovascular and systemic diseases. As the presence of diastolic dysfunction is associated with a poor prognostic factor, we consider it important to include the systematic assessment of diastolic function as part of the routine protocol of many diseases, such as in patients with CHF and normal LV ejection fraction, ischaemia, hypertension, hypertrophy or infiltrative cardiomyopathy. In this group of patients the routine assessment of diastolic function by CMR using PC-CMR allows the evaluation of LV filling

Table 1 Causes of diastolic dysfunction

Common causes

Ischaemic cardiomyopathy

Systemic hypertension

Hypertrophic cardiomyopathy

Aortic valve stenosis

Other causes

Infiltrative cardiomyopathies

Amyloidosis

Sarcoidosis

Iron overload cardiomyopathy (primary or secondary)

Other storage diseases

Glycogen storage disease

Anderson-Fabry disease

Mucopolysaccharidosis

Fibroplastic cardiomyopathies

Endomyocardial fibrosis

Endocardial fibroelastosis

Loffler's fibroplastic endocarditis

Pericardial disorders

Constrictive pericarditis

Pericardial effusion and tamponade 
patterns, with only a couple of minutes added to a standard CMR examination time, providing a more comprehensive clinical evaluation of the LV in addition to LV systolic function assessment. Thus, the diastolic function can be accurate and easily evaluated using morphological and quantitative parameters similar to those obtained by TTE, which are so familiar to general cardiologists. Therefore, the increasing role of CMR in the assessment of the cardiovascular system requires a greater awareness and knowledge of this condition by radiologists.

\section{References}

1. Vasan RS, Benjamin EJ (2001) Diastolic heart failure- no time to relax. N Engl J Med 344:56-59

2. Zile M, Brutsaert D (2002) New concepts in diastolic dysfunction and diastolic heart failure: Part I: diagnosis, prognosis, and measurements of diastolic function. Circulation 105:1387-1393

3. Zile MR, Brutsaert DL (2002) New concepts in diastolic dysfunction and diastolic heart failure: Part II: causal mechanisms and treatment. Circulation 105:1503-1508

4. Vasan RS, Benjamin EJ, Levy D (1995) Prevalence, clinical features and prognosis of diastolic heart failure: an epidemiologic perspective. J Am Coll Cardiol 26:1565-1574

5. Owan TE, Hodge DO, Herges RM, Jacobsen SJ, Roger VL, Redfield MM (2006) Trends in prevalence and outcome of heart failure with preserved ejection fraction. N Engl J Med 355:251259

6. Mandinov L, Eberli FR, Seiler C, Hess OM (2000) Diastolic heart failure. Cardiovasc Res 45:813-825

7. Senni M, Redfield MM (2001) Heart failure with preserved systolic function. A different natural history? J Am Coll Cardiol $38: 1277-1282$

8. Wheeldon NM, Clarkson P, MacDonald TM (1994) Diastolic heart failure. Eur Heart J 15:1689-1697

9. Kovacs SJ, Meisner JS, Yellin EL (2000) Modeling of diastole. Cardiol Clin 18:459-487

10. Katz AM, Zile MR (2006) New molecular mechanism in diastolic heart failure. Circulation 113:1922-1925

11. Paulus WJ, Tschöpe C, Sanderson JE, Rusconi C, Flachskampf FA, Rademakers FE et al (2007) How to diagnose diastolic heart failure: a consensus statement on the diagnosis of heart failure with normal left ventricular ejection fraction by the Heart Failure and Echocardiography Associations of the European Society of Cardiology. Eur Heart J 28:2539-2550

12. Angeja BG, Grossman W (2003) Evaluation and management of diastolic heart failure. Circulation 107:659-663

13. Rathi VK, Doyle M, Yamrozik J, Williams RB, Caruppannan K, Truman C et al (2008) Routine evaluation of left ventricular diastolic function by cardiovascular magnetic resonance: a practical approach. J Cardiovasc Magn Reson 10:36

14. Bellenger NG, Burgess MI, Ray SG, Lahiri A, Coats AJ, Cleland JG et al (2000) Comparison of left ventricular ejection fraction and volumes in heart failure by echocardiography, radionuclide ventriculography and cardiovascular magnetic resonance: are they interchangeable? Eur Heart J 21:1387-1396

15. Srichai MB, Lim RP, Wong S, Lee VS (2009) Cardiovascular applications of phase-contrast MRI. AJR Am J Roentgenol 192:662-675
16. Paelinck BP, Lamb HJ, Bax JJ, Van der Wall EE, de Roos A (2002) Assessment of diastolic function by cardiovascular magnetic resonance. Am Heart J 144:198-205

17. Laffon E, Lecesne R, De Ledinghen V, Valli N, Couzigou P, Laurent $F$ et al (1999) Segmented 5 versus nonsegmented flow quantitation: comparison of portal vein flow measurements. Invest Radiol 34:176-180

18. van den Hout RJ, Lamb HJ, van den Aardweg JG, Schot R, Steendijk P, van der Wall EE et al (2003) Real-time MR imaging of aortic flow: influence of breathing on left ventricular stroke volume in chronic obstructive pulmonary disease. Radiology 229:513-519

19. Chatzimavroudis GP, Zhang H, Halliburton SS, Moore JR, Simonetti OP, Schvartzman PR et al (2003) Clinical blood flow quantification with segmented $\mathrm{k}$-space magnetic resonance phase velocity mapping. J Magn Reson Imaging 17:65-71

20. Tang C, Blatter DD, Parker DL (1993) Accuracy of phase-contrast flow measurements in the presence of partial-volume effects. J Magn Reson Imaging 3:377-385

21. de Haan MW, Kouwenhoven M, Kessels AG, van Engelshoven JM (2000) Renal artery blood flow: quantification with breathhold or respiratory triggered phase-contrast MR imaging. Eur Radiol 10:1133-1137

22. Arheden H, Saeed M, Törnqvist E, Lund G, Wendland MF, Higgins CB et al (2001) Accuracy of segmented MR velocity mapping to measure small vessel pulsatile flow in a phantom simulating cardiac motion. J Magn Reson Imaging 13:722-728

23. Hamilton CA, Moran PR, Santago P 2nd, Rajala SA (1994) Effects of intravoxel velocity distributions on the accuracy of the phase-mapping method in phase-contrast MR angiography. J Magn Reson Imaging 4:752-755

24. Sakuma H, Kawada N, Kubo H, Nishide Y, Takano K, Kato N et al (2001) Effect of breath holding on blood flow measurement using fast velocity encoded cine MRI. Magn Reson Med 45:346348

25. Gatehouse PD, Keegan J, Crowe LA, Masood S, Mohiaddin RH, Kreitner KF et al (2005) Applications of phase-contrast flow and velocity imaging in cardiovascular MRI. Eur Radiol 15:21722184

26. Lotz J, Meier C, Leppert A, Galanski A (2002) Cardiovascular flow measurement with phase-contrast MR imaging: basic facts and implementation. Radiographics 22:651-671

27. Caruthers SD, Lin SJ, Brown P, Watkins MP, Williams TA, Lehr $\mathrm{KA}$ et al (2003) Practical value of cardiac magnetic resonance imaging for clinical quantification of aortic valve stenosis: comparison with echocardiography. Circulation 108:2236-2243

28. Chatzimavroudis GP, Oshinski JN, Franch RH, Walker PG, Yoganathan AP, Pettigrew RI (2001) Evaluation of the precision of magnetic resonance phase velocity mapping for blood flow measurements. J Cardiovasc Magn Reson 3:11-19

29. Lin SJ, Brown PA, Watkins MP, Williams TA, Lehr KA, Liu W et al (2004) Quantification of stenotic mitral valve area with magnetic resonance imaging and comparison with Doppler ultrasound. J Am Coll Cardiol 44:133-137

30. Karwatowski SP, Brecker SJ, Yang GZ, Firmin DN, Sutton MS, Underwood SR (1995) Mitral valve flow measured with cine MR velocity mapping in patients with ischemic heart disease: comparison with Doppler echocardiography. J Magn Reson Imaging 5:89-92

31. Lee VS, Spritzer CE, Carroll BA, Pool LG, Bernstein MA, Heinle SK et al (1997) Flow quantification using fast cine phase-contrast MR imaging, conventional cine phase-contrast MR imaging, and Doppler sonography: in vitro and in vivo validation. AJR Am J Roentgenol 169:1125-1131

32. Fyrenius A, Wigström L, Bolger AF, Ebbers T, Ohman KP, Karlsson M et al (1999) Pitfalls in Doppler evaluation of diastolic 
function: insights from 3-dimensional magnetic resonance imaging. J Am Soc Echocardiogr 12:817-826

33. Hoskins PR (1996) Accuracy of maximum velocity estimates made using Doppler ultrasound systems. Br J Radiol 69:172-177

34. Appleton CP, Jensen JL, Hatle LK, Oh JK (1997) Doppler evaluation of left and right ventricular diastolic function: a technical guide for obtaining optimal flow velocity recordings. J Am Soc Echocardiogr 10:271-292

35. Nishimura RA, Tajik J (1997) Evaluation of diastolic filling of left ventricle in health and disease: Doppler echocardiography is the clinician's Rosetta Stone. J Am Coll Cardiol 30:8-18

36. Senni M, Tribouilloy CM, Rodeheffer RJ, Jacobsen SJ, Evans JM, Bailey KR et al (1998) Congestive heart failure in the community: a study of all incident cases in Olmsted County, Minnesota, in 1991. Circulation 98:2282-2289

37. Vasan RS, Larson MG, Benjamin EJ, Evans JC, Reiss CK, Levy D (1999) Congestive heart failure in subjects with normal versus reduced left ventricular ejection fraction: prevalence and mortality in a population-based cohort. J Am Coll Cardiol 33:1948-1955
38. Kitzman DW, Gardin JM, Gottdiener JS, Arnold A, Boineau R, Aurigemma $G$ et al (2001) Importance of heart failure with preserved systolic function in patients $>$ or $=65$ years of age. CHS Research Group. Cardiovascular Health Study. Am J Cardiol $87: 413-419$

39. Aurigemma GP, Gottdiener JS, Shemanski L, Gardin J, Kitzman D (2001) Predictive value of systolic and diastolic function for incident congestive heart failure in the elderly: the cardiovascular health study. J Am Coll Cardiol 37:1042-1048

40. Dauterman KW, Massie BM, Gheorghiade M (1998) Heart failure associated with preserved systolic function: a common and costly clinical entity. Am Heart J 135:S310-S319

41. O'Connor CM, Gattis WA, Shaw L, Cuffe MS, Califf RM (2000) Clinical characteristics and long-term outcomes of patients with heart failure and preserved systolic function. Am J Cardiol $86: 863-867$

42. Galderisi M (2005) Diastolic dysfunction and diastolic heart failure: diagnostic, prognostic and therapeutic aspects. Cardiovasc Ultrasound 3:9 